\title{
What is the value of an observable between pre- and postselection?
}

\author{
Lars M. Johansen \\ Department of Technology, Buskerud University College, N-3601 Kongsberg, \\ Norway
}

\begin{abstract}
Hall's recent derivation of an exact uncertainty relation [Phys. Rev. A64, 052103 (2001)] is revisited. It is found that the Bayes estimator of an observable between pre- and postselection equals the real part of the weak value. The quadratic loss function equals the expectation of the squared imaginary part of the weak value.
\end{abstract}

Key words: Weak values, exact uncertainty relation, Bayes estimator, loss function PACS: 03.65.Ta, 02.50.-r, 02.50.Tt

Hall recently solved the problem of finding the most efficient estimator for an observable on basis of a measurement of another, incompatible observable [1]. As a consequence, he also found an "exact uncertainty relation". In this short note, we revisit Hall's derivation and provide a new interpretation of the results. In particular, we demonstrate that the weak value of an observable is the most efficient estimator of an observable between preselection and postselection. This result is consistent with the fact that weak values are measured in weak measurements where the interaction between the system and the measurement apparatus is weak [2]. Also, we show that the loss function, which expresses the uncertainty in the estimate, equals the squared imaginary part of the weak value. This result is closely related to the exact uncertainty relation [1].

It was demonstrated by Aharonov et al. [2] that if a "weak measurement" is performed of an observable $\hat{a}$ between preselection of a state $|\psi\rangle$ and postselection of a state $|b\rangle$, then what is measured is the "weak value" of the observable,

$$
a_{w}(b)=\frac{\langle b|\hat{a}| \psi\rangle}{\langle b \mid \psi\rangle} .
$$

Email address: lars.m.johansen@hibu.no (Lars M. Johansen).

Preprint submitted to Elsevier Science 
More precisely, the expectation value of the meter reading equals the real part of the weak value. It has also been shown that the imaginary part of the weak value can be observed in a weak measurement [3]. $a_{w}$ has also been investigated in other settings. Thus, the real part of $a_{w}$ has been interpreted as the "local value" of the observable $\hat{a}[4,5,6,7]$.

In recent years, weak values and weak measurements have been applied to a variety of problems in quantum mechanics. For example, there has been a long discussion on what is the correct way of calculating the time spent by a particle in a tunneling region. Steinberg [8] has pointed out that in the tunneling time problem we are actually dealing with a pre- and postselected system. Steinberg also proposed to use weak values in determining the tunneling time. Weak values have been applied in the interpretation of the double slit experiment [9]. It has been shown that weak values apply to fiber optical networks [10]. Weak values have also been observed in quantum optical experiments $[11,12]$. Recently, it was proposed to test Hardy's paradox [13] experimentally through weak measurements [14]. This suggestion has aroused a lot of interest [15].

Following Hall [1], we use the strategy of finding the best possible guess of $\hat{a}$, i.e., the best possible estimator. In quantum mechanics, observables are stochastic quantities. In standard parametric estimation theory, the aim is usually to estimate a fixed, but unknown parameter. It is therefore not very well suited to solving our problem. On the other hand, Bayesian estimation theory is designed for the estimation of stochastic parameters. It can also take into account prior information about the parameter. We shall consider the preselected state as prior information about the observable. We consider in general a mixed state $\hat{\rho}$ as prior.

The most efficient estimator, or the Bayes estimator, is defined as the estimator that minimizes a given loss function. The loss gives a measure of the deviation between the estimator and the intrinsic observable to be estimated. There are several different loss functions in use in Bayesian estimation theory. The quadratic loss is most frequently used, and can be compared with the prevalence of variance as the most frequently used measure of spread around an expectation value.

We introduce $\hat{\theta}$ as an estimator for $\hat{a}$. As usual in estimation theory, this estimator must be a function of the measurement, i.e., the postselection operator $\hat{b}$. Therefore, these operators commute, $[\hat{\theta}, \hat{b}]=0$.

The quadratic loss is

$$
L(\hat{\theta})=\left\langle(\hat{\theta}-\hat{a})^{2}\right\rangle
$$

where $\langle\hat{O}\rangle=\operatorname{Tr} \hat{\rho} \hat{O}$. Next, we introduce the following operator compatible 
with $\hat{b}$

$$
\hat{\alpha}=\int d b \alpha(b)|b\rangle\langle b|
$$

where

$$
\alpha(b)=\frac{\langle b|\hat{a} \hat{\rho}| b\rangle}{\langle b|\hat{\rho}| b\rangle} .
$$

We write $\hat{\alpha}=\hat{\mu}+i \hat{\sigma}$, where

$$
\begin{aligned}
& \hat{\mu}=\frac{1}{2}\left(\hat{\alpha}+\hat{\alpha}^{\dagger}\right)=\int d b \mu(b)|b\rangle\langle b|, \\
& \hat{\sigma}=\frac{1}{2 i}\left(\hat{\alpha}-\hat{\alpha}^{\dagger}\right)=\int d b \sigma(b)|b\rangle\langle b| .
\end{aligned}
$$

It follows that

$$
\begin{aligned}
\mu(b) & =\frac{1}{2}\left[\alpha(b)+\alpha^{*}(b)\right], \\
\sigma(b) & =\frac{1}{2 i}\left[\alpha(b)-\alpha^{*}(b)\right] .
\end{aligned}
$$

The loss can be written in the form [1]

$$
L(\hat{\theta})=\left\langle\hat{a}^{2}\right\rangle-\left\langle\hat{\theta}^{2}\right\rangle+\left\langle(\hat{\theta}-\hat{\mu})^{2}\right\rangle .
$$

It is minimized for the estimator $\hat{\theta}=\hat{\mu}$. This is the Bayes estimator, and it can be shown to be unbiased, $\langle\hat{\mu}\rangle=\langle\hat{a}\rangle$.

Hall has proposed to interpret $\hat{\mu}$ as the "classical component" of the observable $\hat{a}[1]$ (see also $[16,17]$ ). However, this terminology is not very appropriate. If we restrict the attention to pure states $\hat{\rho}=|\psi\rangle\langle\psi|$, then we see that $\mu(b)=$ $\operatorname{Re} a_{w}(b)$. In other words, the Bayes estimator equals the real part of the weak value. Weak values are notoriously known for their bizarre, and sometimes nonclassical properties. For example, the weak value of kinetic energy may be negative [18]. It is therefore hardly justifiable to interpret the Bayes estimator as the "classical component" of the observable.

In Ref. [6] $\mu(b)$ was interpreted as the "local value" of the observable $\hat{a}$, and $\sigma^{2}(b)$ was interpreted as the "local variance". The latter claim was criticized in Ref. [7].

The loss gives a measure of how well the estimator approaches the intrinsic observable $\hat{a}$. We now find a lower bound on the loss. We follow Hall [1] and 
introduce the vectors $|\mu\rangle=\hat{\rho}^{1 / 2} \hat{a}|b\rangle$ and $|\nu\rangle=\hat{\rho}^{1 / 2}|b\rangle$. By using the Schwarz inequality $|\langle\mu \mid \nu\rangle|^{2} \leq\langle\mu \mid \mu\rangle\langle\nu \mid \nu\rangle$, we find that

$$
\left\langle\hat{\alpha}^{2}\right\rangle=\left\langle\hat{\mu}^{2}\right\rangle+\left\langle\hat{\sigma}^{2}\right\rangle \leq\left\langle\hat{a}^{2}\right\rangle
$$

Equality is reached for pure states [1]. From Eq. (9) follows the inequality

$$
L(\hat{\theta}) \geq\left\langle\hat{a}^{2}\right\rangle-\left\langle\hat{\mu}^{2}\right\rangle
$$

The lower bound is obtained for the Bayes estimator $\hat{\mu}$. Combining Eqs. (10) and (11) yields the inequality

$$
L(\hat{\theta}) \geq\left\langle\hat{\sigma}^{2}\right\rangle
$$

The loss is bounded from below by the squared imaginary part of the weak value. The lower bound is attained by the Bayes estimator on pure states. The equality which is attained for the Bayes estimator can be rewritten as an exact uncertainty relation in the case when $\hat{a}=\hat{p}$ and $\hat{b}=\hat{q}[1]$. So, the exact uncertainty relation can be interpreted as saying that the weak value is the Bayes estimator on a pre- and postselected ensemble.

To answer the question posed in the title, we see that a quantum observable has no definite value between pre- and postselection. Even the most efficient estimator has a nonvanishing loss function. However, in the special case when

$\left\langle\hat{\sigma}^{2}\right\rangle$ vanishes, there exists an exact estimate. This requires that $\sigma(b)$, the imaginary part of the weak value, must vanish.

In conclusion, we have shown that the Bayes estimator on a pre- and postselected ensemble is equal to the real part of the weak value. This result agrees nicely with the fact that weak values are observed in weak measurements on pre- and postselected ensembles. We found that a lower bound on the quadratic loss is given by the expectation of the squared imaginary part of the weak value. The bound is reached for the Bayes estimator on pure states.

\section{Acknowledgments}

This work was supported by a grant from Buskerud University College. 


\section{References}

[1] M. J. W. Hall, Exact uncertainty relations, Phys. Rev. A 64 (2001) 052103-1052103-10.

[2] Y. Aharonov, D. Z. Albert, L. Vaidman, How the result of a measurement of a component of the spin of a spin- $\frac{1}{2}$ particle can turn out to be 100, Phys. Rev. Lett. 60 (14) (1988) 1351-1354.

[3] Y. Aharonov, L. Vaidman, Properties of a quantum system during the time interval between two measurements, Phys. Rev. A 41 (1) (1990) 11.

[4] K. K. Wan, P. J. Sumner, Quantum potential and the spatial distribution of observable values, Phys. Lett. A 128 (9) (1988) 458-462.

[5] P. R. Holland, The Quantum Theory of Motion, Cambridge University Press, Cambridge, 1993.

[6] L. Cohen, Local values in quantum mechanics, Phys. Lett. A 212 (1996) 315319 .

[7] J. G. Muga, J. P. Palao, R. Sala, Average local values and local variances in quantum mechanics, Phys. Lett. A 238 (2-3) (1998) 90-94.

[8] A. M. Steinberg, How much time does a tunneling particle spend in the barrier region, Phys. Rev. Lett. 74 (13) (1995) 2405-2409.

[9] H. M. Wiseman, Directly observing momentum transfer in twin-slit "whichway" experiments, Phys. Lett. A 311 (2003) 285-291.

[10] N. Brunner, A. Acin, D. Collins, N. Gisin, V. Scarani, Optical telecom networks as weak quantum measurements with post-selection, quant-ph/0306108.

[11] N. W. M. Ritchie, J. G. Story, R. G. Hulet, Realization of a measurement of a "weak value", Phys. Rev. Lett. 66 (9) (1991) 1107-1110.

[12] A. D. Parks, D. W. Cullin, D. C. Stoudt, Observation and measurement of an optical aharonov-albert-vaidman effect, Proc. R. Soc. Lond. A 454 (1998) 2997-3008.

[13] L. Hardy, Quantum mechanics, local realistic theories, and lorentz-invariant theories, Phys. Rev. Lett. 68 (20) (1992) 2981-2984.

[14] Y. Aharonov, A. Botero, S. Popescu, B. Reznik, J. Tollaksen, Revisiting hardy's paradox: counterfactual statements, real measurements, entanglement and weak values, Phys. Lett. A 301 (2002) 130-138.

[15] Curioser and curioser, New Scientist 178 (2394) (2003) 28.

[16] S. Luo, Statistics of local value in quantum mechanics, Int. J. Theor. Phys. 41 (9) (2002) 1713-1730. 
[17] A. Luis, Phase-space distributions and the classical component of quantum observables, Phys. Rev. A 67 (2003) 064101.

[18] Y. Aharonov, S. Popescu, D. Rohrlich, L. Vaidman, Measurements, error, and negative kinetic energy, Phys. Rev. A 48 (6) (1993) 4084-90. 